\title{
Purpose of the Creation of Man: Quran's True Version of Anthropic Principle
}

\author{
JAVED JAMIL ${ }^{1}$
}

\begin{abstract}
Scientists, especially theoretical physicists and cosmological philosophers have always wondered the beauty of the universe, especially how it has led to the creation or evolution of intelligent beings like us. There is a certain, wondrous beauty in the underlying plan. This question has become the topic of an interesting debate: What is our place in the Universe and was the Universe made for us? These debates have more often than not been influenced by the dominant philosophies of life in the contemporary world, often pushed by the dominant political and economic forces. As the last few centuries have been dominated by the atheistic or agnostic philosophies, Anthropic Principle has been defined in a way suitable for the forces. Quran, the Holy Book of Islam, on the other hand, presents a different version of the questions related to Anthropic Principle, which describes a specific purpose of the creation of man, his relationship with God and the Universe. This has been named "True Anthropic Principle" by the author of this paper.
\end{abstract}

Keywords: anthropic principle, creation of man, Islam, purpose of creation, the Quran

It appears that there is a set of fundamental physical constants that are such that had they been very slightly different, the universe would have been void of intelligent life. This led to the beginning of the debate on what became known as Anthropic Principle. In the major part of the Twentieth Century, the success of science in understanding the macroscopic, microscopic and cosmological worlds led to the strong belief that it is possible to form an entirely scientific explanation of any feature of the Universe. The dominant principle was the Copernican Principle, the idea that no scientific theory should invoke a special place or aspect to humans (Gingerich 1985). Things started changing when our understanding of physics and biology started unveiling a unique specialness to our Universe, regarding the existence of intelligent life (abysss.uoregon.edu).

There are several constants associated with the Laws of Nature. These include the gravitational constant, the speed of light, the electric charge, the mass of the electron, and Planck's constant. If these constants even slightly change, there will be a radical change in the resulting universe. These have been summed up in an article, entitled, The Anthropic Principle as follows:

1. "Gravitational constant: If lower, then stars would have insufficient pressure to overcome Coulomb barrier to start thermonuclear fusion (i.e. stars would not shine). If higher, stars burn too fast, use up fuel before life has a chance to evolve. (The Coulomb barrier is the energy barrier due to electrostatic interaction that two nuclei need to overcome so they can get close enough to undergo a nuclear reaction.)

${ }^{1}$ Javed Jamil, Ph.D., is Head of Chair in Islamic Studies \& Research, Yenepoya University, Mangaluru, KARNTAKA, India, email: doctorforu123@yahoo.com.

https://doi.org/10.24035/ijit.16.2019.005 
2. Strong force coupling constant: Holds particles together in nucleus of atom. If weaker then multi-proton particles would not hold together, hydrogen would be the only element in the Universe. If stronger, all elements lighter than iron would be rare. Also, radioactive decay would be less, which heats core of Earth.

3. Electromagnetic coupling constant: Determines strength of electromagnetic force that couples' electrons to nucleus. If less, then no electrons held in orbit. If stronger, electrons will not bond with other atoms. Either way, no molecules."

All the above constants are critical to the formation of the basic building blocks of life. The range of possible values for these constants is only about 1 to $5 \%$. Outside this range, life (in particular, intelligent life) would be impossible to emerge."

The writer has asked three important questions: 1 . Why is there any structure at all to the Universe; 2. Why does this structure lead to the capability for life to exist, and 3. Why does life lead too intelligence to understand this structure?". So, the fact emerges that the universe is fine-tuned to allow the emergence of the intelligent beings. If this is so, there are three possibilities:

1. There exists one possible Universe 'designed' with the goal of generating and sustaining 'observers'; or,

2. The Universe is a fluke, a random object and we are just lucky it turned out with the right conditions.

3. The Universe is not fine-tuned; quite the opposite is the case, life conditions are very rare, but life is robust. Plus, there can be other forms of life besides carbon-based life.

4. An ensemble of other different universes is necessary for the existence of our Universe (multiple universes) (abysss.uoregon.edu).

So, the writer concludes, "the philosophical dilemma is that the constants of the Universe on a microscopic (atomic constants), macroscopic (electromagnetic forces) and cosmological levels all appear to be extremely fine-tuned in order for life and intelligence to evolve." (abysss.uoregon.edu).

\section{Types of Anthropic Principle}

Let us try to understand more about the various versions of Anthropic Principle. John D. Barrow and Frank Tipler, explained the Strong Anthropic Principle (SAP) stating that the universe is in some sense compelled to eventually have conscious and sapient life emerge within it. Brandon Carter argued in favour of a Weak Anthropic Principle (WAP) which states that the universe's ostensible fine tuning is the result of a type of selection for universes that are habitable by life: i.e., only in a universe capable of eventually supporting life will there be living beings capable of observing and reflecting on the matter (Merriam Webster Online Dictionary).

The Weak rather than Strong principle earned more favour from the Scientific community because the Strong Principle can ultimately convince people about the inevitability of God as the Creator and the Lord of the Universe, something which is contrary to the philosophy of the current World Order dominated by the forces of politics and economics; that cannot tolerate God in Sciences because it could lose their grip on the minds of the people and the change in their attitudes could cause huge loss to their economic and political designs.

Dicke (1961) argued that the age of the universe, as seen by the living observers, cannot be random. Instead, he argued that we are living in a "golden age", because biological factors constrain the universe to be more or less in an age which is neither too young nor too old. Dicke (1961) later reasoned that the density of matter in the universe must be almost exactly the critical density needed

https://doi.org/10.24035/ijit.16.2019.005 
to prevent the Big Crunch. (The Big Crunch is a hypothetical concept about the ultimate fate of the universe, which means that instead of continuing to expand, expansion being the most believed concept, the reverse will happen leading to the collapse of the entire universe to a point). The scientists have calculated that the observed density of baryonic matter, and some theoretical predictions of the amount of dark matter, account for about $30 \%$ of this critical density, with the rest contributed by a cosmological constant. According to Weinberg (1987), the cosmological constant has a remarkably low value, some 120 orders of magnitude smaller than the value particle physics predicts and according to him this is the "worst prediction in physics". If the cosmological constant were only several orders of magnitude larger than its observed value, the universe would suffer catastrophic inflation. This would have precluded the formation of star systems and life would not have emerged.

Strong Anthropic principle (SAP) has been defined differently by different people. Barrow and Tipler (1997) defined it as follows: "The Universe must have those properties which allow life to develop within it at some stage in its history." Unlike the case with Carter's SAP, the "must" is an imperative in this definition. Barrow and Tipler (1986) give three possible elaborations:

1. There exists one possible Universe 'designed' with the goal of generating and sustaining 'observers'.

2. Observers are necessary to bring the Universe into being.

3. An ensemble of other different universes is necessary for the existence of our Universe.

The first one can be seen as simply the classic design argument restated in the garb of contemporary cosmology. It implies that to give rise to intelligent life is the very purpose of the universe, with the laws of nature and their fundamental physical constants set to ensure that a specific form of life like ours will emerge and evolve. According to Barrow and Tipler the second is a valid conclusion from quantum mechanics. On the other hand, Carter merely says that an ensemble of universes is necessary for the Strong Anthropic Principle to count as an explanation.

\section{Quran's Position: True Anthropic Principle}

Anthropic Principle, especially the Strong Anthropic Principle has come closer to what has been the established position of Islam since the time of Prophet Muhammad. There is a significant difference though. As scientists have vowed to keep God out of the arena of Sciences, they have given an atheistic definition of Anthropic Principles. It will be explained below in detail as to why there has been such an antagonism towards accepting the role of a Designer behind the wonderful Design and the Planner behind the Grand Plan that the Universe obviously is. Though Strong Anthropic Principle almost leads to a theistic conclusion, its proponents too have not dared to say it in straight words. True Version of Anthropic Principle can be developed by understanding what Quran says:

And We did not create the heaven and the earth and that between them aimlessly. That is the assumption of those who disbelieve. (Sad 38:27)

I did not create Jinn and Man for anything but worshipping Me. (al-Dhariyyat 51:56)

Worshipping does not merely mean the ritual of worship; it means total commitment to God's System of laws, reverence and wholehearted devotion. Anthropic Principles regard man as the desired Product of the Creation. But Quran clarifies that: 
1. The creation of the universe was not coincidental but was a well-designed predetermined act of God;

2. God controlled the whole process of creation, creating the planet earth in a unique way, and it ultimately resulted into the creation of the best product, the human being;

3. The purpose of the creation of the intelligent being was not merely his ability to admire the beauty of the universe but to realize that such a wondrous, well designed, well-articulated, complex and huge, yet perfectly coordinated system of the universe could not have come into existence, developed and survived without an all-knowing and All-Powerful God who understood in advance, in fact planned, every single Law of the Universe and fixed their various constants and implemented these laws and then follow his commands in the worldly life as intelligent and conscious beings rather than the inherent and unconscious way in which all the components of the Universe follow the Divine, the so-called "Laws of Nature"

4. Similar to the final creation of an intelligent being, the social conditions on the earth were also fine-tuned to bring out the Prophets in accordance with the Divine Principle of Causality ultimately leading to the emergence of the Final Prophet. Man was given freedom but was directed to submit to the System of existence perfected through His Ambassadors and Testaments and govern the Earth in accordance with the Divine commands.

5. The universe will ultimately cease to function, coming to standstill, not due to gradual process of degeneration but because of the command of God. Then another universe will be created with different sets of Physical laws, and all the human beings that existed on the earth will be resuscitated in a newer form and will face reward or punishment for their deeds in the previous world.

Let me explain it further. God created the universe, and first of all made Physical laws for its governance. Each and every particle and each and every corner of the space that formed the universe submitted to the Physical Laws of God. It was simply not in their power to act otherwise. Having created the Physical universe, God intended to create a kind of existence that will not just submit to God because of the inability to act otherwise. This creation will have the capability if it chooses to even disregard the Social Laws of God. (The components in his body will follow the physical and biological laws of God, though.) The submission as well as rejection by this new creation would of course carry more weight than the inherent submission by the physical world, which will make him the most privileged of all the creations. Greater reward and punishment would await it, as it would willingly accept or reject the System of God rather than as part of its in-built nature. To bring this product, God first created biological laws and then spiritual ones. Once man was created and started multiplying, social laws were created and recreated in accordance with the demands of the developing world. Mankind as a whole was the best product of the creation, as it was capable of admiring the beauty of the universe, analysing its functioning and using its parts for its own benefit. But the Best of the best would be those who would recognise the biggest truth of the Universe-that it has been created and is governed by a Supreme Being-and would be His representatives in the world. The messengers came with the books of Guidance in their areas of influence ultimately paving the way for the arrival of the Final Product of Creation who started the process of unification of the whole mankind under the Final System of God. Muhammad (PBUH) came and showed to the world the Way, confirming the truth that was revealed before him and inviting all to the Final version of the Truth. God says in Quran:

Does man calculate that he will be left aimless? (al-Qiyamah 75:36).

https://doi.org/10.24035/ijit.16.2019.005 
Listen! Your Lord said to the Executive (Angels): "I am about to appoint a viceroy on the Earth." (al-Baqarah 2:30).

Moreover, it is He who has made you (man) the viceroy of the Earth; and has given you relative superiority over one another in ranks (in diverse areas) so that He may examine you all in accordance with what He has granted to you. Of course, your Lord is prompt in (declaring) the Results; and surely, He is Pardoning-Merciful (al-An'am 6:165).

The unity of God was aimed at the unity of mankind, and if the mankind fails to unite in its submission to One System, the ultimate purpose of the unity will not be achieved. The duty to achieve this is man's and if it fails it will be held responsible. God has however taken upon Him to help him fulfil this Duty by sending guidelines and models for the mankind. Quran has numerous verses describing the creative power of God and giving hints about the various processes of creation:

God! None is worthy of worship, other than Him, the Living-Eternal. Neither slumber nor sleep seizes Him. His is whatever is there in the Cosmic Tiers and the Earth. Is there any who can interpose in His presence until He signals? He knows what is ahead of them (in time and space) and what is behind them. They cannot encompass any piece of His Knowledge except what He causes. His Seat extends all over the Cosmic Tiers and the Earth and He is never exhausted in regulating them; further, He is HighGreat (al-Baqarah 2:255).

He is God; none is worthy of worship other than Him, the one with absolute Knowledge about the Incomprehensible and the Comprehensible; He is KindMerciful. He is God; none is worthy of worship other than Him; Emperor-HolyPerfect-Source-of-Peace-Monitor-Powerful-Authoritative-Commanding. The Supreme Force is God's; what then makes them indulge in polytheism! He is God, Creator-Inventor-Designer. The Best Titles are His sole monopoly. Every entity in the Cosmic Tiers and the Earth moves glorifying Him; and He is Powerful-Wise (al-Hashr 59:22-24).

Whatever is there in the Cosmic Tiers and the Earth, along with their fields, rotates, willingly or unwillingly, in glorification of God, causing morning and evening (al-Ra'd 13:15).

Old translations use "shadows", which in fact appear to mean physical fields like electromagnetic and gravitational fields. All the planetary bodies have gravitational fields which extend to long distances depending upon the sizes and masses of these bodies. These fields, which are like extensions of the effects of the bodies, move along with the bodies.

Sajdah, usually translated as bowing or prostration, in terms of Physics may indicate the rotation on one's own axis. As human existence has physical, biological, spiritual and social components, his sajdah is also comprehensive. Not physically able to rotate in continuation, he moves as much as he can. The existence of physical entities, from stars to atomic particles, is physical, so their sajdah is also physical, their rotation being in respect to and in compliance of God who encompasses the whole universe. 
His (God's) wonders include the night and the day, and the Sun and the Moon; if you wish to continue to exclusively worship Him, do never prostrate to the Sun or the Moon; (instead) prostrate to God alone who created the two (Fussilat 41:37).

Can't you observe that it is in respect to God that everything in the Cosmic Tiers and the Earth - the Sun and the Moon, the stars, the mountains, the trees, the animals and a large number of human beings -- rotates (in Compliance to His Laws)? (al-Hajj 22:18).

Every single particle within atom is rotating and so do the stars, planets and satellites. Along with them everything rotates. Human beings committed to God, in addition, do prostrate consciously too.

The Sun is not allowed to break the law in order to capture the Moon, and the Night does not supersede the Day: each one floats in (its own) orbit (Yasin 36:40).

The Seven Cosmic tiers and the Earth and every entity in between revolve glorifying Him; there is nothing that does not revolve (to glorify Him) along with His Acknowledgement; but the truth is that you are unable to explore their Movement of Glorification; God in Indeed Lenient-Pardoner (al-Isra' 17:44).

Every single action requires one or the other kind of physical movement. Like Sajdah, Tasbih, usually translated as glorification, may also mean a specific movement in terms of Physics. Derived from Sabaha (running or floating in a cycles) it implies revolution, which apparently appears to be around another object (like Earth revolving round Sun) but is in fact again in respect to God encompassing the universe. If for example one moves parallel to the outer boundary of a round shaped ground, he will appear to be also moving around the centre. Human tasbih is again physical, biological, spiritual as well as social, while that of the physical entities is only physical. When humans rehearse tasbih, they make movements of tongues or hands using biological energy and do so for the pleasure of God. Stars and other bodies just revolve physically around another object, which ultimately is the part of the whole universe moving to acknowledge God.

Whatever is in the Cosmic Tiers and the Earth revolves glorifying God, Emperor-HolyPowerful-Wise (al-Jumu'ah 62:1).

The State of Cosmic Tiers and Earth is His; and all the carriers of laws move toward Him (al-Hadid 57:5).

Umur represent the fundamental laws of nature. These include gravity and the two nuclear forces, the Strong and the Weak. These along with light (electromagnetic) form what is now known as the Four Fundamental Forces of Nature. These forces are responsible for the functioning of the universe. There may be other forces not yet discovered.

Revered is the One in whose hands is the State of (Universe), and He has authority over everything. (He is) the One who created Death and Life so that He may examine who among you is excellent in performance (al-Mulk 67:1-2). 
It is clear from Quran that the universe is not just a space containing individual entities in the form of stars, planets and moons but is a well-developed and highly organized system governed, sustained and regulated by God. (This writer has identified the Seven Heavens of Quran in terms of Astrophysics with the Atmosphere and Gravitational Field of the Earth being the First Cosmic Tier, the Solar System the Second, the Milky Way (Galaxy) the 3rd, the Local Group (Cluster) the fourth, the Supercluster the fifth, the Distant layer of the universe containing Quasars as the Sixth and the Periphery of the Universe as the Seventh Cosmic Tier (Javed 2019) Quran talks of universe not as something having no entity of its own, having no dynamic existence, but as one that is acting as a fully grown administrative set-up like that of a country. It describes the universe as "Mulk" or State and God as its "Malik" or "Head". After studying Quran, it can be noted without an iota of uncertainty that:

1. The universe is a well-developed state like an administrative setup with a Head;

2. The universe has a well-established constitution comprising various laws and forces like Electromagnetic Force, Gravitational Force, Laws related to Quantum mechanics, laws related to heat, light, electricity, etc;

3. The universe has well established Power centres and offices like Lawh Mahfuz (Secure Tablet), Bayt al-Ma'mur, Arsh, etc;

4. The universe has an advanced system of communication with "umur" (Fundamental Laws and forces), "Executive-Angels", and "Spirit" taking important parts.

5. The universe has a dedicated team of State servants in angels (Executives) (Which may in fact be certain programmers) that perform the duties assigned to them.

6. The universe is composed of several tiers of organization that are called "samawat" or heavens.

7. The universe has been created in a predestined to maintain Order on a long-term basis and create and evolve living beings.

8. Earth is an important part of the universe, where man is acting as Viceroy of the Head of the universe.

For the above system to work there should be a well-established system of Physical laws in position that must have:

1. Vehicles of high enough speed to keep the universe intact, orderly and organized.

2. A mechanism by which the universe does not slip into an ever-increasing entropy.

3. A mechanism that can explain the maintenance of universe in such a low entropy state.

The universe, in short, is a well-organised perfectly coordinated and regulated system like a state with a Lord, God of course being the unchallenged King, President, Governor or Chief Commander of the State of the Universe.

\section{Role of God in Modern Cosmological Philosophies}

From time immemorial man has talked of God. Most of the humans have believed that God created the universe and sustains it. A minuscule percentage of humans have argued that man created, or invented God and their psychological and social needs sustain Him. The debate between what we understand as Sciences and Religion today - the two concepts attained their current understanding only in the last 2-3 centuries - has been quite vociferous. But what was missed altogether was the impact of the emerging philosophies in Political and Economic domains on the dichotomy between 
the two. The evolution of knowledge including natural sciences in the last two centuries has been under the influence of what this writer has called "Economic Fundamentalism". Industrial Revolution resulted in progressive strengthening of the grip of the industrialists over the world and the ideology they propagated.

The new economic philosophy has an overwhelming impact on almost every other philosophy, political, social, legal or religious. Scientific philosophies were not spared either. The impact of the economic fundamentalism on the growth and form of sciences is one of the issues that need a threadbare analysis. The two leading economic theories of the recent times, Capitalism and Socialism and their variants have all been primarily anti-God and anti-religion, though in varying degrees, and their virtual control over all the international institutions including scientific philosophy has led to the predetermined positioning of a "scientific" philosophy regarding God and Creation, which has in fact absolutely nothing scientific about it. Both capitalism and socialism, the two great faces of economic fundamentalism had anathema for God whose fear and love created "unnecessary" impact on human "morals." It was therefore accepted as a fundamental principle by scientists all over the world that God must be kept out of science at all costs. Heisenberg (1959) confirms this when he says:

The mechanics of Newton and all the other parts of the classical physics constructed after its model started from the assumption that one can describe the world without speaking about God or ourselves. This possibility soon seemed almost a necessary condition for natural sciences to grow.

Why should natural sciences start on "the assumption that one can describe the world without speaking about God", if there was no urgency to disprove God? Speaking about "ourselves" might have rightly been an impediment because it would make things more subjective rather than objective. Moreover, "we" made an appearance only recently in the long history of the universe. But why deny God? Had God's existence been accepted, what harm could it have done to sciences? Still, sciences could have tried to understand "God's mind" and His creation and the laws that governed the universe. The only plausible reason behind this position may be that this would have weakened the position of the economic fundamentalists against religion, which (with the exception of only a few religions like Buddhism and Jainism that appear to be agnostic if not atheistic) had belief in God as the foundation on which it rested. Religion posed huge risks to the advance of the economic designs of the forces of economic fundamentalism. Religion promoted morality, abstinence from certain practices like alcohol, gambling, extramarital sex, homosexuality and simplicity in life. All these positions were seen as the foes of "development", and religion therefore was not acceptable. Faith in God and His punishment to the evildoers would greatly reduce the speed of the "growth".

It is said that Sciences do not accept anything unless it is experimentally proved. This in itself is an unscientific principle because the existence of anything, material, law or anything else, is not dependent on its proof by experiments. Experiments continue to grow in quality and quantity and what was beyond the experimental reasoning three centuries ago is within its reach now. This process will go on. If a few centuries back, we had no idea of supernova, quasars or planets outside Solar system, it did not prove that they do not exist. If even today there are no experimental proofs of a highly intelligent life elsewhere in the universe, it does not prove that they are not there. The truth is that we are still in a very early stage of exploring; depending on experiments alone is highly irrational. Even greater is the truth that we are limited by certain other factors which do not allow us to see the present status of the phenomena and objects particularly in the distant world. Experimentally, there is no way we can prove what was exactly there in the universe around 10000 or 1 million or 1 million years back. We can only theorise on the basis of what we can observe today, 
the laws we have discovered and the properties we have studied. None of these is beyond revision. Our knowledge after 300 years will be vastly different from what it is today in the 21st Century. Experimentally, it was not proved around 1000 years back that Alcohol causes Cirrhosis or Promiscuity and Homosexuality are open invitations for sex transmitted diseases. That would not prove that they were safe practices from the medical point of view. Furthermore, there are physical constraints, the biggest one being the speed of light, which may appear to be fast from our standards, but is a mere crawl in terms of the vastness of the universe. We are in no position to observe the distant past of the nearby objects and the present of the distant objects.

Further, the major philosophies about creation in the modern world are mere conjectures and have become popular only because they suit the game plan of the forces. The two most sought out and most debated theories - Creation of the Universe and Organic Evolution are nothing but a predetermined, motivated and preposterous attempt to prove that the universe and the life evolved on their own. This is another matter that this was clearly the violation of the Cause and Effect the humans understand from very early times and is a universally accepted law of Physics today. But as the forces ruling the world would not like to see the Creation and Evolution guided by some God or Supernatural Being, they immediately jumped to popularise these theories as the biggest revolutions in the field of Sciences. Darwin and Einstein became gods of science as their theories (even if they were not atheists themselves) created a room for a self-evolved universe.

In spite of the general antipathy in the scientific community towards religion and God, sciences could never get free of God altogether. Top scientists couldn't keep away from talking of God. Einstein and Bohr had constant debates about the role of God in the formation and functioning of the universe. In response to the idea of uncertainty that Quantum Mechanics advanced, Einstein, in the now famous duel with Bohr, remarked, "God does not play dice". To this Bohr retorted, "Don't try to tell God what to do!"11 The creation of the universe automatically warranted such discussion. Still, every attempt was made to prove that there was no need of God in the creation of the universe and evolution of living beings.

Scientists have always wondered at the beauty of the universe, especially how it has led to the creation or evolution of intelligent beings like us. There is a certain beauty in the underlying plan. Polkinghorne (1998) says:

The universe, in its rationale, beauty and transparency, looks like a world shot through with signs of mind, and maybe, it's the "capital M" Mind of God we are seeing ... there is some deep-seated relationship between the reason within (the rationality of our minds - in this case mathematics) and the reason without (the rational order and structure of the physical world around us). The two fit together like a glove.

Hawking (1988) says, "The remarkable fact is that the values of these numbers seem to have been very finely adjusted to make possible the development of life." (A Brief History of Time, p 138) Isn't this strikingly intriguing in itself that on the one hand Hawking talks of "striking coincidences" and on the other of "the remarkable fact... that the values of these numbers seem to have been very finely adjusted to make possible the development of life"? Hawking further says, "If the rate of expansion one second after the Big Bang had been smaller by even one part in a hundred thousand million, the universe would have collapsed before it ever reached its present size."

The truth is that the "striking coincidences" and "fine adjustments" cannot go together and are opposite to one another. This is like saying that some people, not knowing one another, randomly threw thousands of stones and all of them fell in a way that a fine straight road got formed. And mind it, the living being is a much more developed and complicated structure than a road. The striking 
coincidences that led to the formation of intelligent life have been briefly summed up on a website, St John in Wilderness: Physics and Faith:

Elements up to Lithium-7 were produced in the Big Bang. All heavier elements were made later inside stars. Hence all of us are "star-stuff". Most of the molecules making up our bodies using elements manufactured in an earlier generation of stars that enriched the interstellar medium through their stellar winds or when they died in supernovae. Our own solar system then formed from this enriched interstellar medium, which contained the elements necessary for life.... However, the synthesis of the heavier elements is difficult -- the only reason they are produced at all is the extraordinary coincidence that carbon has an energy level that is nearly the same as the energies of three alpha particles (helium nuclei) inside a star. This correspondence allows the reaction: three Helium-4 nuclei colliding to form one carbon-12 nuclei ( $\left.3{ }^{4} \mathrm{He}--------->{ }^{12} \mathrm{C}\right)$ to occur with a high enough probability that a reasonable amount of carbon can be made, and from carbon, still heavier elements. (Physicists say the "cross-section" for the process is resonant, which is a consequence of the matching of the energy levels).

Paul Dirac (1902-1984), one of the founders of quantum mechanics, noted that very large dimensionless numbers often arise in particle physics and cosmology. For example, ratio of electrostatic force/gravitational force between a proton and electron $=0.23 \times 10^{40}$; ratio of cosmological distance horizon ("radius of the universe") and "classic electron radius" $=3.7 \times 10^{40}$. It can be shown from the physics of stars that these large ratios are required for the lifetime of the average star to be in the range of billions of years. The rate of expansion of the universe is to be such that several generations of stars have time to age that is, the laws of physics and the initial conditions of the universe seemed "tuned" to allowing several generations of stars to live and die (a requirement for the production and dissemination of the heavier elements). The lifetime of an average star has to be sufficiently long to potentially allow a process such as the evolution of life to occur.

Hawking (1988) describes the extraordinary combination of coincidences as follows:

For example, if the electric charge of the electron had been very slightly different, stars either would have been unable to burn Hydrogen and Helium or else would not have exploded. Of course, there might be other forms of intelligent life, not dreamed of even by writers of science fiction, that did not require the light of star like the Sun or the heavier chemical elements that are made in stars and are flying back into space when the stars explode. Nevertheless, it seems clear that there are relatively less ranges of values for the numbers that would allow the development of any form of intelligent life. Most sets of values would give rise to universes that, although they might be very beautiful, would contain no one able to wonder at that beauty. One can take this either as evidence of a divine purpose in Creation and the choices of the laws of science or as support of the strong Anthropic principle.

But even the arguments of strong and weak Anthropic Principle have been dismissed by those who do not want to see any Designer behind all this design. They try to explain this on the basis of random selections. Again, this is difficult to understand why there is insistence on finding a solution 
without God when a solution with God deals problems much more easily. For example, scientists try to argue that as a result of coincidences and accidents, random selections can occur repeatedly in a way that it can lead to evolution of a better and more intelligent life. But they are not ready to accept that more than the probability of finding innumerable number of such coincidences in a way that they lead to what is desirable, the more probable is the presence of a Being who is designing this. This is like assuming numerous coincidences that led to the making of car rather than accepting that it has been designed and manufactured by a company.

It is also entirely incomprehensible why Occam's Razor (Duignan n.d.) is also disregarded while discussing the role of God. According to the well-known scientific principle, "Pluralitas non est ponenda sine neccesitate". This means the number of entities required for explaining anything must be kept at minimum. If there are many ways to explain something, the easiest and straightest one should be preferred. If there are many roads to reach a specific point, the straightest one should be used. This principle was described by a mediaeval philosopher, Occam of Razor, and is still regarded a strong principle in all sciences. Why then is this principle forgotten when we find that the easiest way to describe the creation and evolution of the universe and intelligent life within it is to accept the presence of an All-Knowing, All-Powerful, Wise God.

If the current theories of the creation of the universe are believed, this is what happened: Immediately after Big Bang, some laws of nature all of a sudden came into force without the presence of any legal expert knowing in advance their subtleness and the implications of their enforcement and without any executive capable of implementing them. These laws initiated the creation of a physical world without any Physicist knowing what was happening and to monitor the progress. There was a very complicated and precise mathematics involved with no mathematician around. Then the chemistry started evolving from Physics with many kinds of molecules forming without any expert of Chemistry. Then after millions and millions of years, Biology started coming into existence as the result of chemical reactions, without any Biologist (Zoologist or Botanist) overseeing the complex system of animal and plant kingdom, their mutual dependence and their dependence on their surroundings. There was huge genetics involved with no one there to even know what Genetics was all about. After around 15 billion years, the first one who would have the intelligence to understand and describe what was going around made his first appearance. Before him, there was nothing in the universe that had any elements capable of thinking, analysing, planning and enforcing. Man, the First Intelligent Being within the Universe, could do nothing but to learn how to survive for next millions of years till about ten thousand years back when he started developing philosophical and sociological principles. And within last 200 years, that intelligent being developed some instruments which could see the universe in significant details. That intelligent being is still crippled in many ways. There are some handicaps, which can become lesser and lesser cumbersome with time to come. There are others, which are more permanent. That intelligent being is totally dependent on the provisions of the universe to understand it and cannot create any one of its own. He has to depend on light, which runs with a tiny speed of $300000 \mathrm{kms}$ per second, a speed with which he cannot even see the Sun till at least 8 minutes have passed. He cannot see any present, whatever he sees is past, even if it is a tiny fraction of a second past. It can see only the near past of the nearby objects and distant past of distant objects. Now, this intelligent being, which got intelligence to try to understand the subtleties of a hugely complex universe, claims of becoming legal expert, physicist, biologist and sociologist who can tell everything about what happened and what is happening in the universe. Poor little chap.

What is more intriguing is the fact that if the Einstein's Light-speed barrier is accepted, it means that the whole creation and the evolution of the universe leading to the evolution of intelligent beings in one or few places of the universe occurred without any effective, fast enough 
communication between the distant components of the universe, as no communication can be faster than light, and light-speed can be called nothing more than a crawl in the background of the vastness of the universe. This will be discussed in detail later.

What further complicates the discussion for the believers of "Coincidence" theories is that the biological extension of Anthropic Principle would even be more difficult for them to explain. There is huge designing in the formation of cell with so many complexities working under great coordination, there is tremendous synthesis of atoms in the cells, there is tremendous coordination between the cells of the same organ, even more wonderful cooperation between different organs and an unbelievable synchronization in the functioning of different system. Cardiovascular, digestive, Nervous, Urogenital, Respiratory, Hormonal and Musculoskeletal System are all dependent on one another in their functioning, which involve numerous complex phenomena. According to estimates, there are more than 7 billons of atoms (that's a 7 followed by 27 zeros) (Dove n.d.) and more than 35 trillion cells. This is also true for the Plant System. And then there is wondrous collaboration between Animal Kingdom and Plant Kingdom; and they both are in perfect synchronization with the Matter and Atmosphere of the Planet Earth. There are an estimated number of around 2.5 million species of animals and 1 million species of plants. All these species appear to have had reached an agreement in advance that animals will supply carbon dioxide to plants and in return plants will supply them nutrition and oxygen. Saying all this is happening without a supreme system of Governance with an Ultimate Head can be called nothing less than a lunatic supposition.

In conclusion, according to the modern day philosophers propelled by a world order designed and coordinated by certain forces, we are part of a universe, which requires huge intelligence (of intelligent beings) and highly advanced tools to be understood but required no intelligence (or Being), none with knowledge in advance about what will be effect of the laws and the matter being created and no tools to be created and governed. What a wonderful conclusion.

Quranic Version of Anthropic Principle provides the only plausible explanation of all that led to the emergence of the Physical as well as Biological Worlds ultimately leading to the Best Product of the Creation, the Intelligent Human Being. This intelligent being has no option but to follow the Social System created by God through His Messengers the same way as the physical components of his body and its surroundings, near and far, follow in precision the Divine Laws of Nature. If the components of the universe had not followed with utmost precision the Divine Laws of Nature, the universe could not have survived. If man does not follow the Divine laws of human life as individuals and as collective existence, it may be called nothing but a pre-determined effort to refute the truth due to either failure in the realization of the Truth under the influence of some misleading theories or due to the vested interests of some individuals or forces. It will lead to, is in fact already moving towards chaos.

\section{References}

Barrow, John D. 1997. Anthropic definitions. Quarterly Journal of the Royal Astronomical Society. 24: 146-53.

Barrow, John D. and Frank J. Tipler, 1986. The Anthropic Cosmological Principle. Oxford, UK; New York, NY: Oxford Univ. Press.

Dicke, R. H. 1961. Dirac's cosmology and Mach's principle. Nature 192(4801): 440-441.

Dove, L. n.d. How many atoms are in a person? How Stuff Works? https://science.howstuffworks.com/atoms-in-person.htm. Retrieved: July 15, 2019.

Duignan, B. n.d. Occam's Razor. Encyclopedia Britannica. https://www.britannica.com/topic/Occams-razor. Retrieved: July 15, 2019. 
Gingerich, O. 1985. Did Copernicus Owe a Debt to Aristarchus? Journal for the History of Astronomy 16(1): 37-39.

Hawking, S. 1988. A Brief History of Time. New York: Bantam

Heisenberg, W. 1958. Physics and Philosophy. London: George Allen and Unwin.

Javed Jamil. 2019. Exploring the Seven "Heavens" (Sab'a Samaawaat) of Qur'an in Astrophysics. https://www.researchgate.net/publication/333582998_Exploring_the_Seven_Heavens_Sab' a_Samaawaat_of_Qur'an_in_Astrophysics. Retrieved: July 15, 2019.

Merriam-Webster Online Dictionary.

Anthropic

Principle. abyss.uoregon.edu/ js/cosmo/lectures/lec24.html. Retrieved: July 15, 2019.

New World Encyclopedia. Anthropic Principle. https://www.newworldencyclopedia.org/entry/Anthropic_principle. Retrieved: July 15, 2019.

Polkinghorne, J. 1998. Science and Theology: An Introduction. London: Fortress Press.

St John in Wilderness. Physics and Faith Physics and Faith 3. Rumors of a Designer, Creator and Sustainer Part II. Quantum Cosmology. The Anthropic Principle. http://www.stjohnadulted.org/sc_trueb.htm. Retrieved: July 15, 2019.

Weinberg, S. 1987. Anthropic bound on the cosmological constant. Physical Review Letters 59(22): 2607-2610. 